\title{
Bronchial hyperreactivity: pathogenesis and treatment options
}

\author{
Martina Antosova ${ }^{1}$, Anna Strapkova ${ }^{1}$, Jana Plevkova ${ }^{2}$ \\ ${ }^{1}$ Comenius University in Bratislava, Jessenius School of Medicine in Martin, Department of Pharmacology, Martin, Slovakia; \\ ${ }^{2}$ Comenius University in Bratislava, Jessenius School of Medicine in Martin, Department of Pathophysiology, Martin, Slovakia. \\ Email: antosova@jfmed.uniba.sk; iplevkova@gmail.com
}

Received 21 July 2011; revised 14 September 2011; accepted 12 October 2011.

\begin{abstract}
This review is written to summarize and critically analyze pathogenesis of bronchial hyperreactivity (BHR) as an underlying outcome for suitable treatment options. It describes and discusses the role of genetic predisposition, inflammatory mediators and other endogenous factors (growth factors, nuclear transcription factors), neural regulation and proinflammatory neurotransmitter in the pathogenesis of BHR. Based on these data it provides brief insight into the treatment options, which could be applied to minimize symptoms of respiratory diseases characterized by BHR and successfully diminish the pathogenesis pathways involved.
\end{abstract}

Keywords: Airway Hyperreactivity; Inflammatory Mediators; Nervous System; Therapeutic Targets

\section{INTRODUCTION}

Main feature of bronchial hyperreactivity (BHR) is inappropriate reaction of the airway smooth muscle to different kind of endogenous or exogenous stimuli [1]. It is critical feature of the bronchial asthma, chronic obstructive pulmonary disease (COPD), upper airway infections, rhinitis, or gastro oesophageal reflux disease. BHR could be caused by the action of many direct or indirect stimuli; such are allergens, chemical irritants, cold air, hypoxia, virus infections or relevant pharmacologically active substances. Epidemiological studies have identified several risk factors recruited into the onset and progress of BHR, such as atopy, sex, age, smoking, change of the pulmonary functions etc. Also different kind of cells have been identified as important in the pathogenesis of the BHR (epithelial cells, immune cells, neurons, airway smooth muscle cells) by the mean of production and release of different inflammatory mediators. The group of mediators being recognized as the most important comprises histamine, leukotrienes, prostaglandins, adenosine, acetylcholine, substance P, neurokinin A and nitric oxide (NO) [2].

\section{RISK FACTORS FOR DEVELOPMENT OF BRONCHIAL HYPERREACTIVITY}

The main risk factors in the pathogenesis of the BHR are genetic predisposition, atopy, age, gender, smoking and inhalation of chemical irritants. It had been identified that BHR is more common in subjects with allergic rhinitis and patients who are sensitive to pollen and house dust. However, not all atopic subjects had been diagnosed as having it, and in turn, not all BHR patients do reveal the symptoms of allergy [3].

The role of the gender in the pathogenesis of BHR is not completely clear, but there are some papers reporting two times higher prevalence of the BHR in boys than in girls. Later the distribution of the BHR between the genders seems to be equal, and finally, the prevalence after the puberty is more frequent in males than in females [4]. Another risk factor for the onset and development of BHR is smoking. Cigarette smoke and other exogenous irritants induce the inflammation of the airways, often accompanied by the epithelial damage thus reducing the barrier for potential irritants. The penetration of the irritants can be accompanied by their reduced enzymatic degradation with release of endogenous mediators, neurotransmitters and changes of the epithelium produced NO levels [5]. Although the smoking is provably related to the pathogenesis of BHR, it's role is surprisingly less important comparing to the atopy [1].

\section{CLASSIFICATION OF BHR}

BHR is classified according the presence of respiratory symptoms as temporary or permanent. Temporary BHR is a phenomenon accompanying inflammatory processes in the upper and lower airways. It is characterized by the dry unproductive cough lasting more than one month. Lung function tests and the laboratory parameters are usually normal. Permanent BHR is usually diagnosed in subjects with allergic rhinitis $(10 \%-50 \%$ of subjects reveal BHR), chronic bronchitis (50\% of subjects) and bronchial asthma (100\% of subjects); however it could be diagnosed also in $10 \%-20 \%$ of otherwise healthy 
people [6].

BHR could be inherited, or acquired. Postma et al. [7] reported that the chromosomal locus $5 \mathrm{q} 31-\mathrm{q} 33$ is responsible for the atopy, but also for the BHR. It was reported that this locus is critical for the onset and development of the airway inflammation, and pathogenesis of bronchial asthma. Acquired BHR usually lack this genetic background and it is a consequence of other disease.

The role of the $\operatorname{IgE}$ pathway in the pathogenesis of BHR defines whether it is specific or nonspecific [8]. Specific hyperreactivity to the allergen is mediated mainly by the IgE, and depends on the mast cells. Nonspecific BHR is IgE independent reaction to methacholine or tendency of the airway smooth muscle to contract after the exposure to stimuli like the cigarette smoke, airborne irritants etc [9].

Regarding the presence of the respiratory symptoms, BHR could be symptomatic or asymptomatic. This classification is based on the fact that approximately $50 \%$ of subjects with BHR do not reveal the respiratory symptoms. Pathogenesis of asymptomatic BHR is not completely understood, however asymptomatic BHR could be strong risk factor for later onset and development of bronchial asthma. It is important to note that more than $60 \%$ of those subjects with asymptomatic hyperreactivity have been clearly assigned by higher atopic score than healthy controls [10].

\section{PATHOGENESIS OF BRONCHIAL HYPERREACTIVITY}

Despite the efforts done to elucidate the entire mechanisms responsible for the onset and development of BHR, this problem is not completely clear. The pathogenesis of BHR is strongly influenced by the type of the underlying pathological process. There are differences in the pathogenesis of BHR in subjects with allergic rhinitis, asthma, or COPD. In each case, the ultimate condition is the contact with the trigger, which initiates neurogenic mechanism with recruitment of neuropeptides, activation of cytokine cascades, inflammatory mediators, reactive oxygen species with simultaneous fail of the antioxidative mechanisms, increases expression of nuclear transcription factors and consecutive airway wall remodelation. Except the action of those mediators, cytokines and chemokines, BHR may be influenced by the changes of the mechanical properties of the airway smooth muscle [11].

\subsection{The Role of Nervous System in Pathogenesis of BHR}

Under the physiological conditions, the tone of the airways smooth muscle is influenced by the cooperation of cholinergic, adrenergic and nonadrenergic-noncholinergic (NANC) systems. NANC is capable of excitatory signalling leading to the muscle contraction (eNANC), and inhibitory signalling leading to the muscle relaxation (iNANC). Dysfunction of the nervous pathways contributes to the pathogenesis of BHR mainly in patients with bronchial asthma.

The main bronchoconstrictory pathway in humans is mediated by the cholinergic signalling. In case of dysfunction of muscarinic M2 receptors and thus increased release of acetylcholine, basal tonus of the smooth muscles may be potentiated. Changes of the M2 receptor action induced by IgE, major basic protein, virus infections, allergens, cytokines or ozone are considered as relevant causes of BRH [2].

Bronchoconstrictory signalling from the vagus nerve is counter balanced by inhibitory sympathetic pathways. Sympathetic adrenergic signalling in the human airway smooth muscle is not well developed, but the $\beta_{2}$ adrenergic receptors of the smooth muscle cells do react to the circulating catecholamine. This signalling leads to the bronchodilatatory response and increase the mucocilliary clearance [12].

Excitatory NANC system is involved into the pathogenesis of the neurogenic inflammation, which is characterized by vasodilatation with a plasma leakage, exudation and increased mucus output, bronchoconstriction and structural changes of the epithelium. Major mediators of eNANC are tachykinins - substance P and neurokinins A and B. Except the neural origin of mentioned molecules they are also released from recruited inflammatory cells such as alveolar macrophages, dendritic cells, eosinophils, lymphocytes and neutrophils.

Inhibitory NANC system is responsible for bronchodilatation, and main molecules involved in iNANC signalling are NO, vasoactive intestinal peptide and neuropeptide $\mathrm{Y}$. The role of the NO in the pathogenesis of BHR is very important, it's action is complex, but the final role of $\mathrm{NO}$ in the pathogenesis of BHR is not completely understood. The bronchodilatatory action of $\mathrm{NO}$ is reduced under the allergic reactions, probably due to release of many inflammatory mediators or reactive oxygen species. Any inflammatory process within the respiratory system may lead to the imbalance at the level of nervous system, thus leading to the bronchial hyperreactivity [2].

\subsection{Inflammatory Cells, Mediators and Nuclear Factors Contributing to BHR}

Number of papers supports the role of inflammation in the pathogenesis of BHR; however, there are more types of inflammation. Eosinophilic inflammation seems to be dominant in subjects with allergic rhinitis and asthma 
while neutrophilic inflammation is more typical for subjects with COPD [1]. The role of inflammatory cells and mediators involved in pathogenesis of BHR is complexity, and their actions are constantly discussed.

The airway epithelium seems to have very important position in this topic. It could synthesize some of the proinflammatory cytokines (IL-1, IL-6, IL-8, TNF- $\alpha$, GM-CSF), which can control growing, differentiation, proliferation and activation of immune cells. The epithelial cells are also the source of some of the bronchoconstrictory or bronchodilatatory factors [6].

Molecule with crucial role in the pathogenesis of BHR is IgE. It has high binding affinity to the receptor FceRI, which is located on the surface of basophiles and mast cells. This reaction between the $\operatorname{IgE}$ and the receptor itself initiates inflammatory cascade with subsequent release of proinflammatory mediators (histamine, interleukins IL-4, IL-5, IL-13, leukotrienes, prostaglandins), thus contributing to the acute and chronic manifestations of respiratory diseases and to the onset and development of BHR [13].

Pathogenesis of BHR is influenced also by the action of Th2 lymphocytes, and mediators produced by them, mainly interleukins IL-4, IL-5 and IL-13, which contribute to the pathogenesis of BHR and activate key effectors cells of the allergic inflammation [14]. IL-13 is supposed to be the key cytokine taking a part in the pathogenesis of BHR, mainly in subjects with allergic asthma. The production of this cytokine is critically increased in atopic and nonatopic asthma and allergic rhinitis. IL-13 mediates some of the very important pathogenetic processes as the increase of IgE production, mucus output, increase of the count and survival of eosinophils, expression of cell adhesion molecules, chemokines etc. In vitro studies have reported that IL-13 can directly influence the airway smooth muscle contraction [15].

Interleukin 4, similarly as the IL-13 reacts to the increased IgE production, and together with the GM-CSF potentiates eosinophilic inflammation [16]. However, the work presented by Kumara a et al. [17] reported that pathogenesis of BHR could be independent on the inflammation and can be present in IL-4 and IL-13 knock-out animals. Inhalation of IL- 5 in experiment leads to the increase of the eosinophils and onset of BHR [18]. IL-9 produced by T lymphocytes, eosinophils and mast cells stimulates in turn proliferation of $\mathrm{T}$ lymphocytes, increases production of $\mathrm{IgE}$ in B lymphocytes and supports proliferation and differentiation of mast cells. IL-9 induces inflammation, mucus output and dramatically increases the airway reactivity [19].

Airway inflammatory processes are influenced also by the tumor necrosis factor- $\alpha$ (TNF- $\alpha$ ), which is released from different kind of immune cells and also epithelium. Increase of the TNF- $\alpha$ level was described in subjects with asthma, COPD and chronic bronchitis. Inhalation of TNF- $\alpha$ in healthy humans increased both airway smooth muscle reactivity and the count of neutrophils in the induced sputum. Except this reaction, TNF- $\alpha$ in subjects with mild asthma increased also the count of eosinophils [20]. Also Th1 released cytokine interferon gammaINF- $\gamma$, participates in the BHR pathogenesis, whereas exact cellular target and sources of INF- $\gamma$ have not been identified. One of the possible sources of INF- $\gamma$ could be CD4 TLy. This hypothesis is supported by the data obtained from CD4 TLy compromised mice, which did not develop BHR in experiment. Some authors consider production of INF- $\gamma$ as a phenomenon, which can modulate contractile response of the airway smooth muscle [21].

Neutrophils and eosinophils contain intracellular stores of digestive enzymes with can directly neutralize microorganisms. Some of these products may be used as markers of the inflammation intensity-for example eosinophilic cationic protein, eosinophilic peroxidase, eosinophilic protein X (EPX) and major basic protein (MBP). Although these proteins may potentiate immune and protective functions, in turn, they are also capable to influence the function of the epithelium and nerve fibres, thus indirectly contributing to the pathogenesis of BHR.

Other molecules contributing to pathogenesis of BHR are GM-CSF, macrophages released inflammatory protein (MIP-1 $\alpha$ ) and monocytes released chemotactic proteins (MCP-1, MCP-2 a MCP-5), which are up regulated in subjects with BHR. Prolonged increase of bronchial reactivity in experiment was induced also by the platelet activating factor-PAF. It acts as a chemotactic factor for eosinophils, and in particular experiments their activation induced epithelial damage, microvascular leakage, submucosal oedema and exudation of the plasma to the airway lumen. In experimental research, there are also other inflammatory mediators which had been tested, like eotaxine, thymus activation and regulated chemokines (TARC), some of the growth factors (TGF- $\beta$, connective tissue growth factor, fibroblasts released growth factors (IGF-1, IGF-2), but their role in the pathogenesis BHR have not been exactly explained [22].

There is also increasing evidence supporting the role of nuclear factors in the pathogenesis of inflammatory processes. One of the most commonly discussed nuclear factors is NF- $\mathrm{kB}$. This factor was for the first time identified in B lymphocytes. It is activated by different kind of stimuli like cytokines (TNF- $\alpha$, IL-1 $\beta$, IL-17), proteinkinase $\mathrm{C}$ activators, (PAF), ROS (hydrogen peroxide, ozone), viruses (cytomegalovirus, influenza virus), anti 
CD-3 antibodies, lipopolysacharides etc. [25]. NF- $\mathrm{KB}$ after activation regulates transcription of different genes, which are important in the immune and inflammatory response, mainly genes encoding proinflammatory cytokines (TNF- $\alpha$, IL-1 $\beta$, IL-2, IL-6 a IL-8; GM-CSF, MC-SF, GC-CSF, chemokines), enzymes synthetizing inflammatory mediators (inducible NO-synthases, cyclooxygenase 2, 5-lipooxygenase, cytosolic phospholipase A2), receptors involved into the immune response (receptors for IL-2) or cell adhesion molecules (ICAM-1, VCAM-1 and E-selectin).

\subsection{Reactive Oxygen Species and Their Role in Pathogenesis of BHR}

Oxidative stress is characterized as an imbalance between the production of reactive oxygen species (ROS) and antioxidative mechanisms. Bronchial reactivity is clearly influenced by the action of the reactive oxygen and nitrogen species. ROS are produced by different types of immune cells including eosinophils, macrophages and mast cells, and epithelial cells, as well [23].

ROS can directly induce epithelial damage, with consecutive increase of bronchoconstrictory response. Superoxide anion reacts with $\mathrm{NO}$ and this reaction is a source of peroxynitrite, which increases bronchial reactivity in vitro and in vivo. Oxidative stress also influences cholinergic proconstrictory signalling, most likely by the oxidative damage of the acetylcholinestherase. ROS commonly activate nuclear transcription factors in the epithelial cells, with release of proinflammatory cytokines, and enhancement of inflammatory and prohyperreactive response. Except this action, ROS are responsible for the lipid peroxidation thus leading to formation of many other mediators with prohyperreactive effects - for example izoprostans, which may modulate the control of airway smooth muscle tone [24].

Oxidative stress may also lead to the proliferation of smooth muscle cells, or epithelial cells, may induce neutrophilic inflammation by the increased expression of IL-8, TNF- $\alpha$, may reduce the action of antiproteases $(\alpha 1$-antitrypsin) and induce apoptosis in epithelial and endothelial cells [25].

As it was mentioned before, oxidative stress is a consequence of imbalance between production of ROS, and antioxidative mechanisms. The main antioxidative systems within the airways are represented by superoxiddismutase (SOD), glutathione peroxidase, glutathione S-transferase and xantin oxidase. Nonenzymatic antioxidative mechanisms involve low molecular weight substances like glutathione, ascorbic acid, uric acid, alpha-tocopherol and bilirubine. Regarding the pathogenesis of the BHR it was identified, that asthmatic subjects have revealed relative lack of SOD or glutathione peroxidase activity [26].

\subsection{Airway Remodelation in Subjects with BHR}

Most of the subjects with prolonged BHR who do not respond to the corticosteroid treatment have chronic structural changes in small airways. These changes involve airway wall thickness, hyperplasic and hypertrophic changes of smooth muscle cells, fibroblasts and goblet cells [27].

Current thinking accredits eosinophils with preeminent contributors to allergic airway responses, including a major role in the development in airway remodelling. However recent data demonstrate that at variance with the prevailing paradigm, eosinophils play negligible role in the generation of house dust mite allergic immunity and airway remodelling [28].

It is very important to identify the connections between immune and structural cells to explain and understand process of remodelation. Immune cells, mainly $\mathrm{Th}_{2}$ lymphocytes, mast cells and eosinophils are capable to produce profibrotic cytokines. In subjects with asthma profibrotic factors are TGF- $\beta$, IL-11, IL-17 and leukotriene $\mathrm{B}_{4}\left(\mathrm{LTB}_{4}\right)$, in subjects with COPD elevation of IL- 8 , TNF- $\alpha$, TGF- $\beta$ a LTB 4 is more common. Less intensive profibrotic action has been ascribed to IL-4 and IL-13. Some of the profibrotic signals may arise from extracellular matrix, epithelial cells or fibroblasts [30]. Other molecules with remodelling potential are endothelin- 1 and $\mathrm{LTC}_{4}$ inducing the expression of the collagen in lung fibroblasts. It was also determined that oncostatine M, a pleiotropic cytokine of the gp 130 cytokine family is implicated in chronic allergic diseases, including airway remodelling [30].

The interaction of two systems - proteases and antiproteases seems to play also important role in subjects with asthma and COPD. Proteases are produced by immune and structural cells, the most known are matrixmetaloproteinases (MMP), which contribute to the degradation of collagen, mainly MMP-2, MMP-3, MMP-8 a MMP-9. Antiprotease activity in this case belongs to the TIMP-1 (tissue inhibitor of MMP), and the imbalance between the MMP-9 and TIMP-1 leads to increase of profibrotic risk. In COPD subjects the proteases are neutrophilic elastase, proteinase 3 , katepsin, $\alpha 1$-antitrypsin, SLPI (secretory leukocyte proteinase inhibitor) and some of the MMP as MMP-1, MMP-2 and MMP-9. Airway remodelation is not ultimately related to the inflammation, sometimes the structural changes at the level of small airways are induced by the changed relationship between epithelial cells and myofibroblasts, when epithelial cells abnormally release TGF- $\beta$ and Th 2 cytokines, what is abnormal, with consecutive proliferation of myofibroblasts [31]. 
The structural changes might be related to the increased activity of arginase. Expression pattern of this enzyme is increased in inflammatory conditions and is under control of proinflammatory cytokines IL-4 and IL-13. It is speculated, that one of the pathomechanisms involved in the onset and development of BHR is a relative deficiency of NO caused just by the increased activity of arginase II and changes of the L-arginine homeostasis. L-arginine is the ultimate substrate for both NOsynthase and arginase [32]. It was documented that long lasting BHR leads to the slow, but progressive airway remodelation, but in turn, slight structural changes induced by mentioned mechanism might be an underlying condition for the onset and development of BHR.

\section{NEW THERAPEUTIC APPROACHES IN THE TREATMENT OF BHR BASED ON PATHOGENETIC BACKGROUND}

General idea of the treatment options in BHR subjects is to minimize the risk factors, and to modulate activity of different mediators involved into the pathogenesis of BHR thus reducing the symptoms, and attenuating underlying pathological processes.

\subsection{Antibodies against Immunoglobulin E (Anti-IgE)}

One of the possibilities how to influence specific hyperreactivity is long lasting application anti-IgE antibodies. Clinical studies reported that such a treatment inhibits not only the early bronchoconstrictory response to the allergen inhalation, but also plays important role in the reduction of the late phase of allergic response. Main representative of this group is omalizumab-recombinant molecule, monoclonal antibody with clinical application for the IgE mediated diseases. Omalizumab binds the $\mathrm{IgE}$ at the site appointed for the receptor. The main action is mediated by the inhibition of the mast cells degranulation, and decrease of the level of circulation interleukins IL-13 and IL-5 [33]. It also reduces free IgE level. It's application significantly reduces the need for corticosteroid, and is safe and effective in the control and management of seasonal allergic rhinitis and allergic asthma [34]. The advantage of the omalizumab is relative lack of side effects, and disadvantage is the high price. The most common side effects of the omalizumab treatment are local reactions, like pain, oedema, erythema, itching, which are restricted to the site of injection. The intensity of most reactions ranges from mild to moderate.

\subsection{Antibodies against Cytokines (Anticytokines)}

There is a lot of cytokines, which may play critical role in the pathogenesis of BHR (IL-4, IL-5 and IL-13). IL-5 is considered to be a key cytokine for the differentiation and accumulation of eosinophils. Biological effect of IL-5 is mediated via specific receptor (IL-5R), which is over expressed in the bronchial mucosa in subjects with atopy, or allergic asthma. Application of specific monoclonal antibodies anti IL-5 significantly reduced the count of eosinophils in the bronchial mucosa and reduced the progression of subepithelial fibrosis; however it did not influence bronchial hyperreactivity $[35,36]$.

Interleukins IL-4 and IL-13 are produced by CD4-Th2 lymphocytes and mast cells. Both groups regulate and support the formation of inflammatory cytokines in macrophages. Overproduction of IL-4 causes hypertrophy of epithelial cells in the trachea and bronchi. Enhanced bronchial reactivity was documented also in the mice with overproduction of IL-4. Corry et al. [38] reported that whereas IL-4 does not influence eosinophilic inflammation, it may significantly reduce the BHR. Similar results were reported after administration of IL-4 receptor antagonists [38].

Over production of IL-13 leads to increase of the count of eosinophils in the airways, which may be also connected with the hypertrophy of epithelial cells, metaplastic changes of goblet cells, subepithelial fibrosis and the onset of bronchial hyperreactivity. Anti-IL-13 used in the experiment reduced the count of intraepithelial eosinophils and immune cells in lamina propria of the airway mucosa, effectively reduced morphologic changes of the goblet cells and partially or completely inhibited BHR [39].

Despite the IL-9 is not considered to be a key mediator in the pathogenesis of BHR, there are some date indicating possible therapeutic application of anti-IL-9 treatment in BHR subjects. The results of studies employing anti-IL-9 documented reduced infiltration of the airway mucosa by eosinophils and lymphocytes, decreased production of IL-4, IL-5 and IL-13, decreased production of macrophages released cytokines and finally decrease of BHR [40].

Macrophage migration inhibitory factor-MIF, which works as an inflammatory cytokine is released in case of allergic inflammation. Antibodies against this cytokine anti-MIF reduced significantly bronchial hyperreactivity induced by allergen challenge, and also reduced the count of immune cells in bronchoalveolar fluid in sensitized animals [41].

Infliximab is also a substance with clinical application. It is a recombinant monoclonal antibody with specific binding site for TNF- $\alpha$, thus reducing its activity. Treatment with infliximab in subjects with persistent asthma reduced the level of TNF- $\alpha$ in sputum, but it did not influence the count of eosinophils in the sputum and 
blood. In general, this treatment reduced the episodes of exacerbation in dose dependent pattern [42]. The effect of infliximab in COPD treatment had not been proven [43]. This drug is effective in the treatment of other inflammatory diseases such are rheumatoid arthritis and Crohn disease [44]. Significant reduction of BHR was reported after the application of antibodies against INF- $\gamma$. This treatment inhibited accumulation of lymphocytes which finally suppressed hyperreactivity by reducing activity of the immune cells action [45].

There are also new data reporting potential therapeutic application of R-phycocyanin, which regulates immune response in mouse, this reducing allergic airway inflammation [46].

\subsection{Selective Phosphodiesterase Inhibitors (PDE)}

Selective phosphodiesterase inhibitors represent the category of drugs with combined bronchodilatatory and anti-inflammatory effects. The most important PDEs in the airways are PDE3 and PDE4, which inhibit degradation of cAMP, thus influencing function of different cell types. Mokry et al. [47] reported the effect of selective PDE3 inhibitor cilostazol and PDE4 inhibitor citalopram on cough and airway reactivity in guinea pigs with allergic airway inflammation. Cilostazol reduced significantly cough induced by inhalation of citric acid in healthy animals, citalopram had the same action in both healthy animals and animals with allergic airway inflammation. Both inhibitors reduced bronchial reactivity to histamine in vitro and in vivo, and then reduced counts of monocytes and neutrophils confirming their anti-inflammatory potential. Another selective inhibitor of PDE3 siguazodan inhibited allergen induced bronchoconstriction in sensitized guinea pigs [48]. The same drug in vitro significantly reduced proliferation of airway smooth muscle [49]. Administration of selective PDE3 inhibitors olprinon [50] and MKS492 [51] induced significant bronchodilatation with minimal systemic side effects.

Inhibition of PDE3 seems to be more accurate target, but inhibitors of PDE4 are used more frequently, as it is documented by the number of studies. Application of selective PDE4 inhibitors (rolipram, roflumilast, cilomilast, piclamilast) significantly reduced the count of immune cells, amount of subepithelial collagen and influenced the epithelial hyperplasia [52]. Rolipram, which belongs to the $1^{\text {st }}$ generation of PDE4 inhibitors, did not meet the criteria for safe drugs, because of serious side effects. The $2^{\text {nd }}$ generations of PDE inhibitors roflumilast and cilomilast have well balanced anti-inflammatory and immunomodulatory action. These drugs also have lower incidence of side effects and seem to be more suitable for clinical use [53]. Application of roflumilast was more effective in subjects with asthma [54], whereas cilomilast was more effective in COPD patients [55]. Lipworth et al [56] published the hypothesis that roflumilast could be as effective as beclometasone dipropionate in the treatment of moderate asthma; therefore it can be an alternative treatment and can replace the inhalative corticosteroids. Selective PDE4 inhibitor CDP840 also reduced TNF- $\alpha$ release from immune cells [57].

\subsection{Inhibition of Transcription Factors}

The inflammatory processes are characterized by increased expression of nuclear transcription factors. The most important factors regarding the BHR are NF- $\mathrm{kB}$ and transcription factor GATA-3 localized in Th2 cytokines. The role of GATA-3 in pathogenesis of BHR is not completely clear, but experimentally induced inhibition of GATA-3 reduced lung inflammation including reduction of eosinophils and Th2 cytokines [58]. Pharmacologic interventions influencing activity of transcription factors may be one of the future therapeutic targets. Corticosteroids are one of the most potent NF- $\mathrm{kB}$ inhibitors and they are actually used for the reduction of BHR. The action of other natural inhibitors like gliotoxin and antioxidants are at the level of basic research, and at the present time, the results of the experiments are sharing conflicting evidence.

\subsection{Utilization of Antioxidants}

It was demonstrated that onset and development of BHR is related to the overproduction of reactive oxygen species and lack of antioxidative mechanisms. Supplementation of antioxidants could be one of the tools how to improve this divergence [59]. Actually available antioxidants with beneficial effect on BHR include snip-trap antioxidants and stable glutathione analogs [60]. Strapkova et al. [61] tested the effect of selected antioxidants on the airway smooth muscle hyperreactivity induced by toluene exposition as a source of reactive forms. Vitamin C and E had been used as a pre-treatment in this experimental set up, however they did not influence the BHR induced by toluene. Also Bielory $\&$ Gandhi [62] reported that supplementation of vitamin $\mathrm{C}$ had positive effect on lung tissue and BHR, however the intensity of inflammation had not been reduced. Administration of mucolytics with antioxidative potential (N-acetylcysteine \& ambroxol) reduced reactivity of airway smooth muscle in toluene induced BHR model [63]. Beneficial effect of N-acetylcysteine had been reported in subjects with fribrotising alveolitis, and this effect is believed to be mediated by increase of glutathione level and thus improvement of antioxidative activity [64]. Other antioxidant selen after three months 
administration in subjects with bronchial asthma did not influence significantly clinical manifestation of the asthma, and did not influence the lung function test, including BHR. From the available data we can assume that impact of antioxidants in BHR is not negligible and better knowledge on the relationships between changed antioxidative status and development of BHR may bring new insight to potential therapeutic application.

\section{SUMMARY}

Bronchial hyperreactivity is a phenomenon which is widely studied in basic experimental conditions, and also in clinical studies. The results of these review reported that bronchial reactivity could be modulated both in positive and negative directions, but there are still a lot of questions which remain to be elucidated in the future.

\section{ACKNOWLEDGEMENTS}

This work was supported by grants: VEGA 1/0010/10, MZ SR 2007/46-UK-11 and Center of Experimental and Clinical Respirology (CEKR II) co-financed from EU sourced.

\section{REFERENCES}

[1] Grootendorst, D.C. and Rabe, K.F. (2004) Mechanisms of bronchial hyperreactivity in asthma and chronic obstructive pulmonary disease. Proceedings of the American Thoracic Society, 1, 77-87. doi: $10.1513 /$ pats. 2306025

[2] Joos, G.F. (2003) Bronchial hyperresponsiveness: Too complex to be useful? Current Opinion in Pharmacology, 3, 233-238. doi:10.1016/S1471-4892(03)00046-8

[3] Riccioni, G., Della Vecchia, R., Castronuovo, M., Di Pietro, V., Spoltore, R., De Benedictis, M., Di Iorio, A., Di Gioacchino, M. and Guagnano, M.T. (2002) Bronchial hyperresponsiveness in adults with seasonal and perennial rhinitis: Is there a link for asthma and rhinitis? International Journal of Immunopathology Pharmacology, 15, 69-74.

[4] Ernst, P., Ghezzo, H. and Becklake, M.R. (2002) Risk factors for bronchial hyperresponsiveness in late childhood and early adolescence. European Respiratory $J_{O}$ urnal, 20, 635-639. doi:10.1183/09031936.02.00962002

[5] Amin, K., Ekberg-Jansson, A., Lofdahl, C.G. and Venge, P. (2003) Relationship between inflammatory cells and structural changes in the lungs of asymptomatic and never smokers: A biopsy study. Thorax, 58, 135-142. doi:10.1136/thorax.58.2.135

[6] Grbac, I., Martinac, S., Šokočevič, M., Rončević, S., Petrič, V. and Stančić, V. (2003) The pathogenesis of bronchial hyperreactivity in patients with allergic rhinitis. Acta Clinica Croatica, 42, 69-74.

[7] Postma, D.S., Kooppelman, G.H. and Meyers, D.A. (2000) The genetics of atopy and airway hyperresponsiveness. American Journal of Respiratory and Critical Care Medicine, 162, S118-S123.

[8] Larche, M., Robinson, D.S. and Kay, A.B. (2003) The role of $\mathrm{T}$ lymphocytes in the pathogenesis of asthma. Journal of Allergy Clinical Immunology, 111, 450-463. doi:10.1067/mai.2003.169

[9] Schmidt, D. (2001) Human isolated airways: An in vitro model for the investigation of airway smooth muscle physiology and its pharmacological modulation. Thesis. Leiden, Leiden University.

[10] Laprise, C. and Boulet, L.P. (1997) Asymptomatic airway hyperresponsiveness: A three-year follow-up. American Journal of Respiratory Critical Care Medicine, 156, 403-409.

[11] Amrani, Y., Moore, P.E., Hoffman, R., Shore, S.A. and Panettieri, R.A. Jr. (2001) Interferon-gamma modulates cysteinyl leukotriene receptor-1 expression and function in human airway myocytes. American Journal of Respiratory Critical Care Medicine, 164, 2098-2101.

[12] Widdicombe, J.G. (1998) Autonomic regulation: iNANC/eNANC. American Journal of Respiratory Critical Care Medicine, 158, S171-S175.

[13] Bardin, P.G., Dorward, M.A., Lampe, F.C., Franke, B. and Holgate, S.T. (1998) Effect of selective phosphodiesterase 3 inhibition on the early and late asthmatic responses to inhaled allergen. British Journal of Clinical Pharmacology, 45, 387-391. doi:10.1046/j.1365-2125.1998.t01-1-00689.x

[14] Barnes, P.J. (2004) Mediators of chronic obstructive pulmonary disease. Pharmacological Reviews, 56, 515-548. doi:10.1124/pr.56.4.2

[15] Barnes, P.J. and Karin, M. (1997) Nuclear factor-B: A pivotal transcription factor in chronic inflammatory diseases. New England Journal of Medicine, 336, 10661071. doi:10.1056/NEJM199704103361506

[16] Yang, M., Hogan, S.P., Henry, P.J., Matthaei, K.I., McKenzie, A.N.J., Young, I.G., Rothenberg, M.E. and Foster, P.S. (2001) Interleukin-13 mediates airways hyperreactivity through the IL-4 receptor-alpha chain and STAT-6 independently of IL-5 and eotaxin. American Journal of Respiratory Cell and Molecular Biology, 25, 522-530.

[17] Bielory, L. and Gandhi, R. (1994) Asthma and vitamin C. Annals of Allergy, 73, 89-96.

[18] Billington, C.K., Joseph, S.K., Swan, C., Scott, M.G., Jobson, T.M. and Hall, I.P. (1999) Modulation of human airway smooth muscle proliferation by type 3 phosphodiesterase inhibition. American Journal of Physiology, 276, L412-419.

[19] Shi, H.Z., Xiao, C.Q., Zhong, D., Qin, S.M., Liu, Y., Liang, G.R., Xu, H., Chen, Y.Q., Long, X.M. and Xie, Z.F. (1998) Effect of inhaled interleukin-5 on airway hyperreactivity and eosinophilia in asthmatics. American Journal of Respiratory Critical Care Medicine, 157, 204-209.

[20] Soussi-Gounni, A., Kontolemos, M. and Hamid, Q. (2001) Role of IL-9 in the pathophysiology of allergic diseases. Journal of Allergy and Clinical Immunology, 107, 575-582. doi:10.1067/mai.2001.114238

[21] Mukhopadhyay, S., Hoidal, J.R. and Mukherjee, T.K. (2006) Role of TNFalpha in pulmonary pathophysiology. Respiratory Research, 11, 125. doi:10.1186/1465-9921-7-125

[22] Thomas, P.S. and Heywood, G. (2002) Effects of inhaled tumour necrosis factor alpha in subjects with mild 
asthma. Thorax, 57, 774-778.

doi:10.1136/thorax.57.9.774

[23] MacNee, W. (2001) Oxidants/antioxidants and chronic obstructive pulmonary disease: Pathogenesis to therapy. Novartis Foundation Symposium, 234, 169-188. doi:10.1002/0470868678.ch11

[24] Spicuzza, L., Barnes, P.J., Di Maria, G.U. and Belvisi, M.G. (2001) Effect of 8-isoprostaglandin F2 on acetylcholine release from paraxympathetic nerves in guinea pig airways. European Journal of Pharmacology, 416, 231-234. doi:10.1016/S0014-2999(01)00882-2

[25] Barnes, P.J. and Karin, M. (1997) Nuclear factor- B: A pivotal transcription factor in chronic inflammatory diseases. New England Journal of Medicine, 336, 10661071. doi:10.1056/NEJM199704103361506

[26] Smith, L.J., Shamsuddin, M., Sporn, P.H., Denenberg, M. and Anderson, J. (1997) Reduced superoxide dismutase in lung cells of patients with asthma. Free Radical Biology and Medicine, 22, 1301-1307. doi:10.1016/S0891-5849(96)00550-3

[27] Boulet, L.P. (2003) Physiopathology of airway hyperresponsiveness. Current Allergy and Asthma Reports, 3, 66-71. doi:10.1007/s11882-003-0030-9

[28] Fattouh, R., Al-Garawi, A., Fattouh, M., Arias, K., Walker, T.D., Goncharova, S., Coyle, A.J., Humbles, A.A. ans Jordana, M. (2011) Eosinophils are dispensable for allergic remodelling and immunity in a model of house dust mite-induced airway disease. American Journal of Respiratory Critical Care Medicine, 183, 179-188. doi:10.1164/rccm.200905-0736OC

[29] Westergren-Thorsson, G., Chakir, J., Lafrenière-Allard, M.J., Boulet, L.P. and Tremblay, G.M. (2002) Correlation between airway responsiveness and proteoglycan production by bronchial fibroblasts from normal and asthmatic subjects. International Journal of Biochemistry and Cell Biology, 34, 1256-1267. doi:10.1016/S1357-2725(02)00058-4

[30] Fritz, D.K., Kerr, C., Fattouh, R., Llop-Guevara, A., Khan, W.I., Jordana, M. and Richards, C.D. (2011) A mouse model of airway disease: Oncostatin M-induced pulmonary eosinophilia, goblet cell hyperplasia, and airway hyperresponsiveness are STAT6 dependent, and interstitial pulmonary fibrosis is STAT6 independent. Journal of Immunology, 186, 1107-1118. doi:10.4049/jimmunol.0903476

[31] Bergeron, C. and Boulet, L.P. (2006) Structural changes in airway diseases: Characteristics, mechanisms, consequences, and pharmacologic modulation. Chest, 129, 1068-1087. doi:10.1378/chest.129.4.1068

[32] Meurs, H., Maarsingh, H. and Zaagsma, J. (2003) Arginase and asthma: Novel insights into nitric oxide homeostasis and airway hyperresponsiveness. Treasury Inflation Protected Securities, 24, 450-455. doi:10.1016/S0165-6147(03)00227-X

[33] Noga, O., Hanf, G. and Kunkel, G. (2003) Immunological and clinical changes in allergic asthmatics following treatment with omalizumab. International Archives of Allergy and Immunology, 131, S46-52. doi:10.1159/000070434

[34] Hamelmann, E. and Wahn, U. (2008) Anti-IgE therapy. Clinical Allergy Immunology, 21, 415-427.

[35] Kumar, R.K., Herbert, C., Thomas, P.S., Wollin, L.,
Beume, R., Yang, M., Webb, D.C. and Foster, P.S. (2003) Inhibition of inflammation and remodelling by roflumilast and dexamethasone in murine chronic asthma. Journal of Pharmacology and Experimental Therapeutics, 307, 349-355. doi:10.1124/ipet.103.053819

[36] Finkelman, F.D., Hogan, S.P., Hershey, G.K., Rotheberg, M.E. and Wills-Karp, M. (2010) Importance of cytokines in murine allergic airway disease and human asthma. Journal of Immunology, 184, 1663-1674. doi:10.4049/jimmunol.0902185

[37] Kotaru, C., Skowronski, M., Coreno, A. and McFadden, E.R. Jr. (2001) Inhibition of nitric oxide synthesis attenuates thermally induced asthma. Journal of Applied Physiology, 91, S703-708.

[38] Tomkinson, A., Duez, C., Cieslewicz, G., Pratt, J.C., Joetham, A., Shanafelt, M.C., Gundel, R. and Gelfand, E.W. (2001) A murine IL-4 receptor antagonist that inhibits IL-4- and IL-13-induced responses prevents antigen-induced airway eosinophilia and airway hyperresponsiveness. Journal of Immunology, 166, 5792-5800.

[39] Lee, C.G., Homer, R.J., Cohn, L., Link, H., Jung, S., Craft, J.E., Graham, B.S., Johnson, T.R. and Elias, J.A. (2002) Transgenic Overexpression of Interleukin (IL)-10 in the lung causes mucus metaplasia, tissue inflammation, and airway remodeling via IL-13-dependent and independent pathways. Journal of Biological Chemistry, 277, 35466-35474. doi:10.1074/jbc.M206395200

[40] Cheng, G., Arima, M., Honda, K., Hirata, H., Eda, F., Yoshida, N., Fukushima, F., Ishii, Y. and Fukuda, T. (2002) Anti-interleukin-9 antibody treatment inhibits airway inflammation and hyperreactivity in mouse asthma model. American Journal of Respiratory and Critical Care Medicine, 166, 409-416. doi:10.1164/rccm.2105079

[41] Amano, T., Nishihira, J. and Miki, I. (2007) Blockade of macrophage migration inhibitory factor (MIF) prevents the antigen-induced response in a murine model of allergic airway inflammation. Inflammation Research, 56, 24-31. doi:10.1007/s00011-007-5184-9

[42] Erin, E.M., Leaker, B.R., Nicholson, G.C., Tan, A.J., Green, L.M., Neighbour, H., Zacharasiewicz, A.S., Turner, J., Barnathan, E.S., Kon, O.M., Barnes, P.J. and Hansel, T.T. (2006) The effects of a monoclonal antibody directed against tumor necrosis factor-alpha in asthma. American Journal of Respiratory and Critical Care Medicine, 174, 753-762. doi:10.1164/rccm.200601-0720C

[43] Rennard, S.I., Fogarty, C., Kelsen, S., Long, W., Ramsdell, J., Allison, J., Mahler, D., Saadeh, C., Siler, T., Snell, P., Korenblat, P., Smith, W., Kaye, M., Mandel, M., Andrews, C., Prabhu, R., Donohue, J.F., Watt, R., Lo, K.H., Schlenker-Herceg, R., Barnathan, E.S. and Murray, J. (2007) COPD Investigators. The safety and efficacy of infliximab in moderate to severe chronic obstructive pulmonary disease. Journal of Respiratory and Critical Care Medicine, 175, 926-934. doi:10.1164/rccm.200607-995OC

[44] Conroy, C.A. and Cattell, R. (2001) Infliximab treatment for Crohn's disease. Postgraduate Medical Journal, 77, 436-440. doi:10.1136/pmj.77.909.436

[45] Kumar, R.K., Herbert, C., Webb, D.C., Li, L. and Foster, P.S. (2004) Effects of anticytokine therapy in a mouse 
model of chronic asthma. American Journal of Respiratory and Critical Care Medicine, 170, 1043-1048. doi:10.1164/rccm.200405-6810C

[46] Chang, C.J., Yang, Y.H., Liang, Y.C., Chiu, C.J., Chu, K.H., Chou, H.N. and Chiang, B.L. (2011) A novel phycobiliprotein alleviates allergic airway inflammation by modulating immune responses. American Journal of Respiratory and Critical Care Medicine, 183, 15-25. doi:10.1164/rccm.201001-0009OC

[47] Mokrý, J. (2010) Inhibítory fosfodiesteráz pri ochoreniach dýchacích ciest. Pediatria Pre Prax, 5, 101-107.

[48] Underwood, D.C., Bochnowicz, S., Osborn, R.R., Kotzer, C.J., Luttmann, M.A., Hay, D.W., Gorycki, P.D., Christensen, S.B. and Torphy, T.J. (1998) Antiasthmatic activity of the second-generation phosphodiesterase 4 (PDE4) inhibitor SB 207499 (Ariflo) in the guinea pig. Journal of Pharmacology and Experimental Therapeutics, 287, 988-995.

[49] Billington, C.K., Joseph, S.K., Swan, C., Scott, M.G., Jobson, T.M. and Hall, I.P. (1999) Modulation of human airway smooth muscle proliferation by type 3 phosphodiesterase inhibition. American Journal of Physiology, 276, L412-419.

[50] Myou, S., Fujimura, M., Kamio, Y., Ishiura, Y., Tachibana, H., Hirose, T., Hashimoto, T. and Matsuda, T. (1999) Bronchodilator effect of inhaled olprinone, a phosphodiesterase 3 inhibitor, in asthmatic patients. American Journal of Respiratory Critical Care Medicine, 160, $817-820$.

[51] Joos, G.F. (2001) The role of neuroeffector mechanisms in the pathogenesis of asthma. Current Allergy and Asthma Reports, 1, 134-143. doi:10.1007/s11882-001-0081-8

[52] Kuss, H., Hoefgen, N., Johanssen, S., Kronbach, T. and Rundfeldt, C. (2003) In vivo efficacy in airway disease models of N-(3,5-dichloropyrid-4-yl)-[1-(4-fluorobenzyl)5-hydroxy-indole-3-yl]-glyoxylic acid amide (AWD 12281), a selective phosphodiesterase 4 inhibitor for inhaled administration. Journal of Pharmacology and Experimental Therapeutics, 307, 373-385. doi:10.1124/jpet.103.053942

[53] Karish, S.B. and Gagnon, J.M. (2006) The potential role of roflumilast: The new phosphodiesterase- 4 inhibitor. The Annals of Pharmacotherapy, 40, 1096-1104. doi:10.1345/aph.1E651
[54] Fernandes, D.J., Mitchell, R.W., Lakser, O., Dowell, M., Stewart, A.G. and Solway, J. (2003) Do inflammatory mediators influence the contribution of airway smooth muscle contraction to airway hyperresponsiveness in asthma? Journal of Applied Physiology, 95, 844-853.

[55] Holgate, S.T. (1998) The inflammation-repair cycle in asthma: The pivotal role of the airway epithelium. Clinical and Experimental Allergy, 5, 97-103. doi:10.1046/j.1365-2222.1998.028s5097.x

[56] Lipworth, B.J. (2005) Phosphodiesterase-4 inhibitors for asthma and chronic obstructive pulmonary disease. Lancet, 365, 167-175. doi:10.1016/S0140-6736(05)17708-3

[57] Essayan, D.M. (2001) Cyclic nucleotide phosphodiesterases. Journal of Allergy and Clinical Immunology, 108, 671-680. doi:10.1067/mai.2001.119555

[58] Finotto, S., De Sanctis, G.T., Lehr, H.A., Herz, U., Buerke, M., Schipp, M., Bartsch, B., Atreya, R., Schmitt, E., Galle, P.R., Renz, H. and Neurath, M.F. (2001) Treatment of allergic airway inflammation and hyperresponsiveness by antisense-induced local blockade of GATA-3 expression. Journal of Experimental Medicine, 193, 1247-1260. doi:10.1084/jem.193.11.1247

[59] Nadeem, A., Raj, H.G. and Chhabra, S.K. (2008) Effect of vitamin E supplementation with standard treatment on oxidant-antioxidant status in chronic obstructive pulmonary disease. Indian Journal of Medical Research, 128, S705-711.

[60] Cuzzocrea, S., Riley, D.P., Caputi, A.P. and Salvemini, D. (2001) Antioxidant therapy: A new pharmacological approach in shock, inflammation, and ischemia/reperfusion injury. Pharmacological Reviews, 53, S135-159.

[61] Strapková, A., Nosálová, G., Adamicová, K. and Giačová, D. (1997) Antioxidants in the respiratory system. Bratisl Lek Listy, 98, 527-530.

[62] Bielory, L. and Gandhi, R. (1994) Asthma and vitamin C. Annals of Allergy, 73, S89-96.

[63] Strapková, A. and Nosálová, G. (2001) Nitric oxide and airway reactivity. Bratisl Lek Listy, 102, S345-350.

[64] Behr, J., Maier, K., Degenkolb, B., Krombach, F. and Vogelmeier, C. (1997) Antioxidative and clinical effects of high-dose $\mathrm{N}$-acetylcysteine in fibrosing alveolitis. Adjunctive therapy to maintenance immunosuppression. American Journal of Respiratory and Critical Care Medicine, 156, S1897-1901. 